\title{
Présentation du texte
}

\section{Anne-Yvonne Julien}

\section{OpenEdition}

\section{Journals}

Édition électronique

URL : http://journals.openedition.org/ccs/880

DOI : $10.4000 /$ ccs. 880

ISSN : 2558-782X

\section{Éditeur :}

Presses universitaires de Rennes, Association des lecteurs de Claude Simon

\section{Édition imprimée}

Date de publication : 31 mars 2013

Pagination : 13-17

ISBN : 9782354121785

ISSN : 1774-9425

\section{Référence électronique}

Anne-Yvonne Julien, «Présentation du texte », Cahiers Claude Simon [En ligne], 8 | 2013, mis en ligne le 21 septembre 2017, consulté le 24 septembre 2020. URL : http://journals.openedition.org/ccs/880

DOl : https://doi.org/10.4000/ccs.880 
Ce texte, "Le Régicide ", qui peut être considéré comme un avant-texte d'un fragment des Géorgiques ${ }^{1}$, a paru dans le numéro de juin-juillet 1977 de La Nouvelle Critique. Cette revue, rappelonsle, dont le premier rédacteur en chef fut Jean Kanapa ${ }^{2}$, créée dans une immédiate proximité avec le Parti communiste d'après-guerre, a d'abord cherché à diffuser auprès d'un public intellectuel les principes de l'analyse marxiste. Mais la revue mensuelle s'ouvre progressivement au débat après février 1956 et la révélation du rapport Khrouchtchev, et plus encore après 1968, sous l'impulsion de Francis $\mathrm{Cohen}^{3}$, directeur et de François Hincker ${ }^{4}$, rédacteur en chef. Son dernier numéro interviendra en 1980. Cette volonté d'ouverture est d'ailleurs clairement affichée par l'Avant-Propos de ce numéro 105 qui propose en couverture le titre "Ecrire " : il s'agit de "'donner à voir' la réalité complexe et contradictoire de la littérature » en acte,

1. Voir à ce sujet l'étude d'Alastair Duncan : «Les textes courts de Claude Simon : tentative de catégorisation ", dans le dossier critique "L'Esthétique des formes brèves dans l'œuvre de Claude Simon ", Cahiers Claude Simon, n7, PUP, 2011, p. 44-59. 2. Jean Kanapa (1921-1978), philosophe de formation, proche de J.-P. Sartre, fondateur et directeur de La Nouvelle Critique entre 1948 et 1967.

3. Francis Cohen (1914-2000), qui fit partie de la direction clandestine de l'UEC (Union des étudiants communistes) pendant la Seconde Guerre, fut journaliste à L'Humanité, directeur de La Nouvelle Critique entre 1967 et 1980 ; il co-signe en collaboration avec A. Adler, M. Décaillot, C. Frioux et R. Léon, L'URSS et nous aux Editions sociales en 1978.

4. On doit notamment à François Hincker l'essai : Le parti communiste au carrefour, essai sur quinze ans de son histoire 1965-1981, éd. Albin Michel, Paris 1981. 
en particulier, en enquêtant sur le " travail d'écriture " de quelques grands contemporains. La rubrique intitulée " Pratiques » est d'ailleurs d'une réelle densité. A côté de la contribution de Claude Simon qui est double, un entretien avec Alain Poirson et Jean-Paul Goux, "Un homme traversé par le travail » (p. 32-44) et un inédit "Le Régicide» (p. 45-46), figurent un article sur l'œuvre poétique d'Aragon, un texte d'Yves Bonnefoy, des extraits de Feuillets d'Hypnos de René Char, un article de Robert Bréchon sur la quête « autobiographique " de Leiris, des fragments du Parti pris des choses et de Proêmes de Françis Ponge, un entretien avec Michel Tournier et un extrait du livre Journal de voyage au Canada que celui-ci était en train d'élaborer en collaboration avec le photographe E. Boubat ${ }^{5}$.

Un certain nombre d'éléments qui figurent dans le texte court se retrouveront quasiment à l'identique dans l'une des séquences de la geste du Conventionnel L. S. M., où est évoqué, hors chronologie, et au filtre de celui qui rêve sur les archives familiales retrouvées, l'épisode de l'ambassade de Naples " au lendemain d'Aboukir "6

5. On citera aussi sur l'axe "Avant-gardes » une discussion entre Jean-Pierre Faye, Claude Prévost, Jean Ricardou et Jean Thibaudeau et un questionnaire, en clôture du numéro, adressé à quelques contemporains sur leur manière de penser le statut d'écrivain, les institutions littéraires et leur propre réflexion critique : voisinent cette fois les signatures d'Emile Ajar, Gaston Baissette, Raphä̈lle Billetdoux, Marie Cardinal, Max Gallo, Raymond Jean, Annie Leclerc, Jean-Patrick Manchette, François Nourissier, André Stil, Claude Ollier, Jules Roy...

6. Qu'il suffise de rappeler que le gouvernement révolutionnaire a déclaré la guerre à l'Angleterre le $1^{\text {er }}$ février 1793 . Nelson est chargé de rétablir la supériorité navale britannique en Méditerranée et il fait route vers les Royaumes de Sardaigne et de Naples pour demander des renforts. Il arrive à Naples au début du mois de septembre où il rencontre Ferdinand I ${ }^{\text {er }}$ des Deux-Siciles. C'est à cette occasion qu'il fera la connaissance d'Emma Hamilton, l'épouse de l'ambassadeur britannique, pour laquelle il se prendra de passion. Nelson reçoit l'ordre fin décembre 93 d'organiser le blocus de la garnison française en Corse. Toulon vient de tomber aux mains des Républicains. Bastia est prise en mai 94 : l'amiral commence le siège de Calvi en juin, la ville capitulera, mais à l'occasion d'un échange de tirs, il sera touché à l'œil droit. Au cours de l'été 98, il s'oppose aux desseins de Bonaparte, alors général en chef, prêt à l'invasion de l'Egypte, en poursuivant la flotte française et en la surprenant au mouillage en rade d'Aboukir le $1^{\text {er }}$ août 1798. C'est effectivement " au lendemain d'Aboukir ", que Nelson revient à Naples en héros. Toutefois Ferdinand $1^{\text {er }}$ n'acceptera de déclarer la guerre à la France qu'en novembre et sous 
( $G$, p. 57-59). Deux composants interviennent toutefois qui ne réapparaissent pas dans les pages concernées du roman : d'une part, un effet de montage d'un fragment du " Journal » de LSM, relatif à une visite aux sites antiques de Pompeya et d'Herculanum en forme de bribes discontinues (avec alternance d'éclats phrastiques en caractères romains et italiques, parfois raturés), d'autre part, le faux-fil d'une réflexion de Michelet empruntée à l'Histoire de la Révolution française, III, Livre X, chapitre $2^{7}$, centrée sur les menées britanniques contre la France révolutionnaire et l'alliance Londres-Naples.

Michelet, dans ce chapitre, trace un portrait au vitriol de William $\operatorname{Pitt}^{8}$, qui se réjouit en secret de la mort du Roi de France et en escompte l'abaissement définitif de la puissance française ; avec le sens superbe de la formule qui est le sien, l'essayiste définit ainsi Pitt: "Une perfection d'homme haïssable et désolante. L'austérité sans la vertu » et il enchaîne : "dans la grande poursuite qui a absorbé sa vie, la ruine de la Révolution, la destruction de la France, il n'a reculé devant nulle chose criminelle, honteuse ou basse. Les révolutionnaires lui ont imputé, je le sais, beaucoup de choses douteuses. Pour s'en tenir au certain, il en reste de quoi effrayer Machiavel ». C'est le contexte immédiatement antérieur de la première phrase citée de Michelet dans le fragment de Simon : "Obligé d'entrer dans le détail curieux, il est vrai, mais malpropre, de la diplomatie (anglaise et européenne), dans l'intérieur triste et sale de cette cuisine politique, on doit prier le lecteur de résister au dégoût. Omnia munda mundis ${ }^{9}$ ". Quant au "Il faut etc., etc. ", il recouvre "Il faut imiter la lumière, qui dans sa pureté supérieure, peut impunément pénétrer dans les

la pression de son épouse Marie-Caroline, sœur de Marie-Antoinette et de ses soutiens à la cour. A la fin de l'été 98, les jeux d'alliance ne sont donc pas encore faits : L. S. M. peut encore espérer peser sur le roi de Naples. C'est de cette fin d'été 98, décisive pour l'équilibre des forces en Méditerranée, qu’il est parlé en termes de " poisseuse puanteur suspendue dans la chaleur de septembre ».

7. Jules Michelet, Histoire de la Révolution française, t. III, Paris, éd. Hetzel, 1883, chapitre intitulé «La coalition. - Meurtre de Basville. 13 janvier 93 », p. 210-211. 8. William Pitt le Jeune (1759-1806), " Premier ministre " de Grande-Bretagne entre 1783 et 1801 et de nouveau entre 1804 et 1806 .

9. "Tout est pur pour ceux qui sont purs ", phrase extraite d'une épître de Paul à Tite, I, 15, devenue formule à visée morale courante. 
lieux les plus immondes ", et ajoute Michelet, " ne se salir nulle part ". De là, l'historien invite tout de même son lecteur à aller voir du côté de l'action délétère de l'Angleterre sur Naples, ce qui génère la seconde affirmation citée à propos de Pitt " il ('Pitt' dans le texte de Simon) y régnait sans conteste : sur le palais, la reine, la chambre à coucher et le lit royal... » Passage où s'amorce la diatribe contre la "Messaline " galloise (diatribe scindée ici en trois bribes) qui n'est autre qu'Emma Hamilton, déshonorant Nelson qu'elle gouvernait à sa guise, en exigeant que " le pavillon britannique servit de potence " aux " chefs prisonniers de la République de Naples " (alliés de la France révolutionnaire).

Reste à faire quelques hypothèses sur les raisons qui ont pu conduire Claude Simon à concéder une autonomie à ce texte court et à lui donner ce support de publication. Commençons peut-être par le second point. On sait que la posture de l'écrivain engagé déplaît à Simon et celui-ci ne manque d'ailleurs pas de le redire à Alain Poirson dans l'entretien paru dans ce même numéro. Il n'empêche que le titre accordé à cette brève prose "Le Régicide " et l'écran historique sur lequel se dessine la silhouette du revenant à la "stature d'Hercule" ne sont pas neutres. Que La Nouvelle Critique qui, depuis les années 70 , se retrempe dans les fondements de l'esprit révolutionnaire, ait pu accueillir cette sélection simonienne n'a en tout cas rien d'étonnant. Et puis, il faudrait aussi souligner la présence citationnelle de Michelet, chantre romantique du peuple, lui-même plus que nourri par les philosophes des Lumières. Mais si pensée de la révolution il y a, elle n'est en rien didactique, elle passe par les associations verbales, les antithèses ou les parallélismes induits : se dit ici le combat entre deux temporalités, celle effectivement de l'action révolutionnaire, de l'impulsivité du verbe, de l'effort oratoire et celle de la réaction, des soubresauts du vieux monde monarchiste, et nul doute que la stagnation de l'été napolitain, les signes de la pourriture portuaire ne s'accordent aux indices d'une décadence ambiante. Quant à la note incertaine d'un tel combat, elle est émise par le lien suggéré entre les surcharges hâtives de l'écriture de l'ancêtre et les «strates de laves » des « cités [latines] rayées » de toute carte. Si maintenant l'on cherche à cerner un régime d'écriture, difficile de ne pas concevoir que ce texte sous-titré " fragments » au pluriel et proposé 
dans les environs immédiates de quelques "Feuillets " de Char et de quelques "Proêmes » de Ponge, accueille sur le mode récurrent l'idée même de la fragmentation (" particules de marbre ", "statues brisées, [...] colonnes ", " poudroiement lumineux ", " ossements, [...] ruines ", " dentelles déchiquetées ", " ratures ", " bavures "...).

On l'aura compris, les pages des Géorgiques (p. 57-59) en résonance avec cet avant-texte ne sont pas marquées par le même degré de discontinuité, puisqu'elles se sont allégées de certains éléments. Ou plutôt, le jeu de discontinuité est ailleurs, dans le montage de ce somptueux chapitre I qui entremêle épisodes divers de la geste de Lacombe Saint-Michel, délibérément déchronologisées, séquences de guerre d'un cavalier pris dans la déroute française en 1940, épreuves d'un jeune engagé britannique auprès des forces du Poum ${ }^{10}$ dans le Barcelone de 36 et instants de fatigue ou d'attention extrême de celui qui rêve à l'ensemble de ces expériences. Il reste que cet épisode napolitain est lesté d'un poids particulier de mélancolie car le décor de ce Naples fin de siècle est propice à la signification tout à la fois de la pugnacité des combats humains et de leur devenir de poussière. Comment ne pas être sensible à la vision étrange de la rade diaprée de lumière que l'auteur prête au regard du Conventionnel, cet effet d'" infinitésimales et scintillantes particules de marbre ", sorte d'émanation des ruines situées alentour qui annonce de près l'expression retenue en clôture de chapitre premier, pour dire le regard du sujet scripteur sur les carnets de l'ancêtre :

«Lorsque l'on tourne les pages des registres en les tenant inclinés de fines particules couleur rouille aux facettes scintillantes et dorées comme du mica se détachent des lettres et glissent sur les feuilles. On dirait que les mots assemblés, les phrases, les traces laissées sur le papier par les mouvements de troupe, les combats, les intrigues, les discours s'écaillent, s'effritent et tombent en poussière, ne laissant plus sur les mains que cette poudre impalpable couleur de sang séché ». $(G$, p. 76)

A.-Y. J.

10. Partido Obrero de Unificacion Marxista. 